\title{
The Effects of Field-Aligned Rotation on the Magnetically Channeled Line-Driven Winds
}

\author{
Asif ud-Doula \\ Department of Physics, North Carolina State University, Raleigh, NC \\ 27695-8202, USA \\ Stanley Owocki \\ Bartol Research Institute, University of Delaware, Newark, DE 19716, \\ $U S A$
}

\begin{abstract}
There is extensive evidence that the radiatively driven stellar winds of OB-type stars are not the steady, smooth outflows envisioned in classical models, but instead exhibit extensive structure and variability on a range of temporal and spatial scales. We examine the possible role of stellar magnetic fields in forming large-scale wind structure. It is based on numerical magnetohydrodynamic (MHD) simulations of the interaction of a line-driven flow with an assumed stellar dipole field.

Unlike previous fixed-field analyses, the MHD simulations here take full account of the dynamical competition between field and flow, and thus apply to a full range of magnetic field strength, and within both closed and open magnetic topologies. A key result is that the overall degree to which the wind is influenced by the field depends largely on a single, dimensionless, 'wind magnetic confinement parameter', $\eta_{*}\left(=B_{e q}^{2} R_{*}^{2} / \dot{M} v_{\infty}\right)$, which characterizes the ratio between magnetic field energy density and kinetic energy density of the wind.

We extend these MHD simulations to include field-aligned stellar rotation. The results indicate that a combination of the magnetic confinement parameter and the rotation rate as a fraction of the 'critical' rotation now determine the global properties of the wind. For models with strong magnetic confinement, rotation can limit the extent of the last closed magnetic loop, and lead to episodic mass ejections that break through the close loop and are carried outward with a slow, dense, equatorial outflow. Our 2-D numerical simulations indicate that the magnetic fields provide excessive amount of angular momentum to the wind preventing the formation of a Keplerian disk.
\end{abstract}

\section{Overview of the Results}

In a recent paper (ud-Doula \& Owocki, ApJ 576, 2002), we have shown that the degree to which line-driven hot-star winds are influenced by the magnetic fields depends largely on a single, dimensionless, 'wind magnetic confinement parameter', $\eta_{*}\left(=B_{e q}^{2} R_{*}^{2} / \dot{M} v_{\infty}\right)$, which characterizes the ratio between magnetic field energy density and kinetic energy density of the wind. Here, we extend our study to include the influence of field-aligned rotation on such winds. 
It is well known that the hot stars are rapid rotators. Numerous spectroscopic studies (e.g. Conti \& Ebbets 1977; Fukuda 1982) indicate that normal OB-type stars are characterized by typical rotational velocities in excess of 100 $\mathrm{km} / \mathrm{s}$, and some are observed to have projected rotational speeds of up to 400 $\mathrm{km} / \mathrm{s}$. As such, it is important that we examine the role of rotation on their winds.

The radiation force is an efficient mechanism to lift the stellar material off the surface, but it is not efficient in providing angular momentum to that material. An efficient mechanism to impart angular momentum to outflowing material is the moment arm of a stellar magnetic field. Our general approach is to study the nature of the wind outflow for various assumed values of the wind magnetic confinement parameter, $\eta_{*}$ and the rotation rate as a fraction of the 'critical' rotation, $w \equiv \Omega / \Omega_{c}$.

We study the competition between the field and wind by evolving our MHD simulations from an initial condition at time $t=0$, when a dipole magnetic field and rigid body stellar rotation is suddenly introduced into a previously relaxed, one-dimensional, spherically symmetric wind. In general, rotation enhances the equatorial density and reduces the equatorial flow velocity.

For small confinements, $\eta_{*} \leq 1$ and rotation $w=1 / 2$ the equatorial density is noticeably enhanced compared to the zero-rotation cases. Higher density leads to slower equatorial outflow. For strong magnetic confinements for which the Alfven radius is smaller than the co-rotation radius, the simulations are similar to the non-rotational cases. The field remains closed in loops near the equatorial surface. Wind outflows accelerated upward from opposite polarity footpoints are guided towards a strong collision near the loop tops. The material that is stagnated as a result of this, is eventually pulled back by gravity onto the star in a complex flow pattern. Unlike, for the strong confinement cases for which the Alfven radius is larger, the stagnated material doesn't reach the co-rotation radius, and thus is not flung away outward.

For strong magnetic confinements for which the Alfven radius is larger than the co-rotation radius, the situation is quite different from the the zero-rotation case. Some of the material that is stagnated within the closed loops and lies above the co-rotation radius, is spun up to speeds such that the outward centrifugal force is larger than the inward pull of gravity. Consequently, this material punches through the field lines and is flung away bullet/ring-like patterns. On the other hand, the material that lies below the co-rotation radius, is pulled back by the gravity.

There is only a very limited spatial range over which the wind material can be launched into a Keplerian orbit. A simple scaling analysis shows that one needs some fine-tuning in the choice of the magnetic fields to obtain a model with enough but not excessive spin to launch the wind material into a Keplerian orbit. However, none of our dynamical simulations form such a disk. Currently, we are carrying out a more extensive set of simulations to see if there might be any range of parameters for which a disk can form.

This work was supported by the NASA Space Grant College program at the University of Delaware, by NASA grants NAG5-3530 and NAG-11095, and by NSF grant AST-0097983. 